\title{
Upper Bound Estimation of Fractal Dimensions of Fractional Integral of Continuous Functions
}

\section{Yongshun Liang}

Faculty of Science, Nanjing University of Science and Technology, Nanjing, China Email: liangyongshun@gmail.com

Received 1 December 2014; revised 15 December 2014; accepted 1 January 2015

Copyright (C) 2015 by author and Scientific Research Publishing Inc.

This work is licensed under the Creative Commons Attribution International License (CC BY). http://creativecommons.org/licenses/by/4.0/

(c) (i) Open Access

\section{Abstract \\ Fractional integral of continuous functions has been discussed in the present paper. If the order of Riemann-Liouville fractional integral is $v$, fractal dimension of Riemann-Liouville fractional inte- gral of any continuous functions on a closed interval is no more than $2-v$.}

\section{Keywords}

\section{Box Dimension, Riemann-Liouville Fractional Calculus, Fractal Function}

\section{Introduction}

In [1], fractional integral of a continuous function of bounded variation on a closed interval has been proved to still be a continuous function of bounded variation. The upper bound of Box dimension of the Weyl-Marchaud fractional derivative of self-affine curves has given in [2]. Previous discussion about fractal dimensions of fractional calculus of certain special functions can be found in [3] [4].

In the present paper, we discuss fractional integral of fractal dimension of any continuous functions on a closed interval.

If $U$ is any non-empty subset of $n$-dimensional Euclidean space, $R^{n}$, the diameter of $U$ is defined as $|U|=\sup \{|x-y|: x, y \in U\}$, i.e. the greatest distance apart of any pair of points in $U$. If $\left\{U_{i}\right\}$ is a countable collection of sets of diameter at most $\delta$ that cover $F$, i.e. $F \subset \bigcup_{i=1}^{\infty} U_{i}$ with $0<\left|U_{i}\right| \leq \delta$ for each $i$, we say that $\left\{U_{i}\right\}$ is a $\delta$-cover of $F$.

Suppose that $F$ is a subset of $R^{n}$ and $s$ is a non-negative number. For any positive number define.

$$
\mathcal{H}_{\delta}^{s}(F)=\inf \left\{\sum_{i=1}^{\infty}\left|U_{i}\right|^{s}:\left\{U_{i}\right\} \text { is a } \delta \text {-cover of } F\right\}
$$


Write

$$
\mathcal{H}^{s}(F)=\lim _{\delta \rightarrow 0} \mathcal{H}_{\delta}^{s}(F)
$$

$\mathcal{H}_{\delta}^{s}(F)$ is called s-dimensional Hausdorff measure of $F$. Hausdorff dimension is defined as follows:

Definition 1.1 [5] Let $F$ be a subset of $R^{n}$ and $s$ is a non-negative number. Hausdorff dimension of $F$ is

$$
\operatorname{dim}_{H}(F)=\inf \left\{s: \mathcal{H}^{s}(F)=0\right\}=\sup \left\{s: \mathcal{H}^{s}(F)=\infty\right\}
$$

If $s=\operatorname{dim}_{H}(F)$, then $\mathcal{H}^{s}(F)$ may be zero or infinite, or may satisfy

$$
0<\mathcal{H}^{s}(F)<\infty
$$

A Borel set satisfying this last condition is called an s-set.

Box dimension is given as follows:

Definition 1.2 [5] Let $F$ be any non-empty bounded subset of $R^{n}$ and let $N_{\delta}(F)$ be the smallest number of sets of diameter at most $\delta$ which can cover $F$. Lower and upper Box dimensions of $F$ respectively are defined as

$$
\underline{\operatorname{dim}}_{B}(F)=\underline{\lim }_{\delta \rightarrow 0} \frac{\log N_{\delta}(F)}{-\log \delta}
$$

and

$$
\overline{\operatorname{dim}}_{B}(F)=\varlimsup_{\overline{\lim }}{ }_{\delta \rightarrow 0} \frac{\log N_{\delta}(F)}{-\log \delta}
$$

If (1.1) and (1.2) are equal, we refer to the common value as Box dimension of $F$

$$
\operatorname{dim}_{B}(F)=\lim _{\delta \rightarrow 0} \frac{\log N_{\delta}(F)}{-\log \delta}
$$

Definition 1.3 [6] Let $f(x) \in C_{[0,1]}$ and $v>0$. For $t \in[0,1]$ we call

$$
D^{-v} f(x)=\frac{1}{\Gamma(v)} \int_{0}^{x}(x-t)^{v-1} f(t) \mathrm{d} t
$$

Riemann-Liouville integral of $f(x)$ of order $v$.

\section{Riemann-Liouville Fractional Integral of 1 -Dimensional Fractal Function}

Let $f(x)$ be a 1-dimensional fractal function on $I$. We will prove that Riemann-Liouville fractional integral of $f(x)$ is bounded on $I$. Box dimension of Riemann-Liouville fractional integral of $f(x)$ will be estimated.

\subsection{Riemann-Liouville Fractional Integral of $f(x)$}

Theorem 2.1 Let $D^{-v} f(x)$ be Riemann-Liouville integral of $f(x)$ of order $v$. Then, $D^{-v} f(x)$ is bounded. Proof. Since $f(x)$ is continuous on a closed interval $I$, there exists a positive constant $M$ such that

$$
|f(x)| \leq M \quad \forall x \in I
$$

From Definition 1.3, we know

$$
D^{-v} f(x)=\frac{1}{\Gamma(v)} \int_{0}^{x}(x-t)^{v-1} f(t) \mathrm{d} t \quad 0<v<1
$$

For any $\quad x \in I$, it holds 


$$
\left|D^{-v} f(x)\right| \leq \frac{M}{v \Gamma(v)} x^{v} \leq \frac{M}{\Gamma(v+1)} \quad 0<v<1
$$

$D^{-v} f(x)$ is a bounded function on $I$.

\subsection{Fractal Dimensions of Riemann-Liouville Fractional Integral of $f(x)$}

Theorem 2.2 Let $D^{-v} f(x)$ be Riemann-Liouville integral of $f(x)$ of order $v$. Then,

$$
1 \leq \operatorname{dim}_{H} \Gamma\left(D^{-v} f, I\right) \leq \overline{\operatorname{dim}}_{B} \Gamma\left(D^{-v} f, I\right) \leq 2-v, \quad 0<v<1
$$

Proof. Let $0<\delta<1 / 2$, and $m$ is the least integer greater than or equal to $1 / \delta$. If $0 \leq a_{1}<b_{1} \leq \delta$, we have

$$
\begin{aligned}
\Gamma(v)\left[D^{-v} f\left(b_{1}\right)-D^{-v} f\left(a_{1}\right)\right] & =\left(\int_{0}^{b_{1}}\left(b_{1}-t\right)^{v-1} f(t) \mathrm{d} t-\int_{0}^{a_{1}}\left(a_{1}-t\right)^{v-1} f(t) \mathrm{d} t\right) \\
& =\int_{0}^{a_{1}}\left[\left(b_{1}-t\right)^{v-1}-\left(a_{1}-t\right)^{v-1}\right] f(t) \mathrm{d} t+\int_{a_{1}}^{b_{1}}\left(b_{1}-t\right)^{v-1} f(t) \mathrm{d} t .
\end{aligned}
$$

For $1 \leq i \leq m$, let $M_{i}=\max _{x \in[(i-1) \delta, i \delta]} f(x), m_{i}=\min _{x \in[(i-1) \delta, i \delta]} f(x) \quad M=\max _{x \in I} f(x)$ If $D^{-v} f\left(b_{1}\right)-D^{-v} f\left(a_{1}\right) \geq 0$, it holds

$$
\Gamma(v+1)\left[D^{-v} f\left(b_{1}\right)-D^{-v} f\left(a_{1}\right)\right] \leq\left(b_{1}-a_{1}\right)^{v}\left(M_{1}-m_{1}\right)+\left(b_{1}^{v}-a_{1}^{v}\right) m_{1}
$$

If $D^{-v} f\left(b_{1}\right)-D^{-v} f\left(a_{1}\right)<0$, it holds

$$
\Gamma(v+1)\left|D^{-v} f\left(b_{1}\right)-D^{-v} f\left(a_{1}\right)\right| \leq\left(b_{1}-a_{1}\right)^{v}\left(M_{1}-m_{1}\right)
$$

We have

$$
\left|D^{-v} f\left(b_{1}\right)-D^{-v} f\left(a_{1}\right)\right| \leq \frac{1}{\Gamma(v+1)}\left(b_{1}-a_{1}\right)^{v}\left(M_{1}-m_{1}\right)+M \delta^{v}
$$

Let $1 \leq n \leq m-1$. If $n \delta \leq a_{n+1} \leq b_{n+1} \leq(n+1) \delta$, we have

$$
\begin{aligned}
\Gamma(v)\left[D^{-v} f\left(b_{n+1}\right)-D^{-v} f\left(a_{n+1}\right)\right]= & \int_{0}^{n \delta+b_{n+1}}\left(n \delta+b_{n+1}-t\right)^{v-1} f(t) \mathrm{d} t-\int_{0}^{n \delta+a_{n+1}}\left(n \delta+a_{n+1}-t\right)^{v-1} f(t) \mathrm{d} t \\
= & \int_{0}^{n \delta}\left[\left(n \delta+b_{n+1}-t\right)^{v-1}-\left(n \delta+a_{n+1}-t\right)^{v-1}\right] f(t) \mathrm{d} t \\
& +\int_{n \delta}^{n \delta+a_{n+1}}\left[\left(n \delta+b_{n+1}-t\right)^{v-1}-\left(n \delta+a_{n+1}-t\right)^{v-1}\right] f(t) \mathrm{d} t \\
& +\int_{n \delta+a_{n+1}}^{n \delta+b_{n+1}}\left(n \delta+b_{n+1}-t\right)^{v-1} f(t) \mathrm{d} t .
\end{aligned}
$$

If $D^{-v} f\left(b_{n+1}\right)-D^{-v} f\left(a_{n+1}\right) \geq 0$, it holds

$$
\Gamma(v+1)\left[D^{-v} f\left(b_{n+1}\right)-D^{-v} f\left(a_{n+1}\right)\right] \leq\left(b_{n+1}-a_{n+1}\right)^{v}\left(M_{n+1}-m_{n+1}\right)+\left(b_{n+1}^{v}-a_{n+1}^{v}\right) m_{n+1}
$$

If $D^{-v} f\left(b_{n+1}\right)-D^{-v} f\left(a_{n+1}\right)<0$, it holds

$$
\Gamma(v+1)\left|D^{-v} f\left(b_{n+1}\right)-D^{-v} f\left(a_{n+1}\right)\right| \leq\left(b_{n+1}-a_{n+1}\right)^{v}\left(M_{n+1}-m_{n+1}\right)+(2 \delta)^{v} M
$$

We get

$$
\left|D^{-v} f\left(b_{n+1}\right)-D^{-v} f\left(a_{n+1}\right)\right| \leq \frac{1}{\Gamma(v+1)}\left(b_{n+1}-a_{n+1}\right)^{v}\left(M_{n+1}-m_{n+1}\right)+2 M \delta^{v}
$$

There exists a positive constant $C$, such that 


$$
R_{D^{-v} f}[i \delta,(i+1) \delta] \leq C \delta^{v}, \quad 1 \leq i \leq m-1
$$

If $N_{\delta}\left(D^{-v} f\right)$ is the number of squares of the $\delta$ mesh that intersects $\Gamma\left(D^{-v} f, I\right)$, by Proposition 11.1 of [1], we have

$$
N_{\delta}\left(D^{-v} f\right) \leq 2 m+\delta^{-1} \sum_{i=0}^{m-1} R_{D^{-v} f}[i \delta,(i+1) \delta] \leq C \delta^{v-2}
$$

From (1.2) of Definition 1.2, we know

$$
\overline{\operatorname{dim}}_{B} \Gamma\left(D^{-v} f, I\right)=\varlimsup_{\delta \rightarrow 0} \frac{\log N_{\delta}\left(D^{-v} f\right)}{-\log \delta} \leq 2-v, \quad 0<v<1
$$

With Definition 1.1, we get the conclusion of Theorem 2.2.

This is the first time to give estimation of fractal dimensions of fractional integral of any continuous function on a closed interval.

\section{Acknowledgements}

Research is supported by NSFA 11201230 and Natural Science Foundation of Jiangsu Province BK2012398.

\section{References}

[1] Liang, Y.S. (2010) Box Dimension of Riemann-Liouville Fractional Integral of Continuous Function of Bounded Variation. Nonlinear Analysis Series A: Theory, Method and Applications, 72, 2758-2761.

[2] Yao, K. and Liang, Y.S. (2010) The Upper Bound of Box Dimension of the Weyl-Marchaud Derivative of Self-Affine Curves. Analysis Theory and Application, 26, 222-227. http://dx.doi.org/10.1007/s10496-010-0222-9

[3] Liang, Y.S. and Su, W.Y. (2011) The Von Koch Curve and Its Fractional Calculus. Acta Mathematic Sinica, Chinese Series [in Chinese], 54, 1-14.

[4] Zhang, Q. and Liang, Y.S. (2012) The Weyl-Marchaud Fractional Derivative of a Type of Self-Affine Functions. Applied Mathematics and Computation, 218, 8695-8701. http://dx.doi.org/10.1016/j.amc.2012.01.077

[5] Falconer, J. (1990) Fractal Geometry: Mathematical Foundations and Applications. John Wiley Sons Inc., New York.

[6] Oldham, K.B. and Spanier, J. (1974) The Fractional Calculus. Academic Press, New York. 
Scientific Research Publishing (SCIRP) is one of the largest Open Access journal publishers. It is currently publishing more than 200 open access, online, peer-reviewed journals covering a wide range of academic disciplines. SCIRP serves the worldwide academic communities and contributes to the progress and application of science with its publication.

Other selected journals from SCIRP are listed as below. Submit your manuscript to us via either submit@scirp.org or Online Submission Portal.
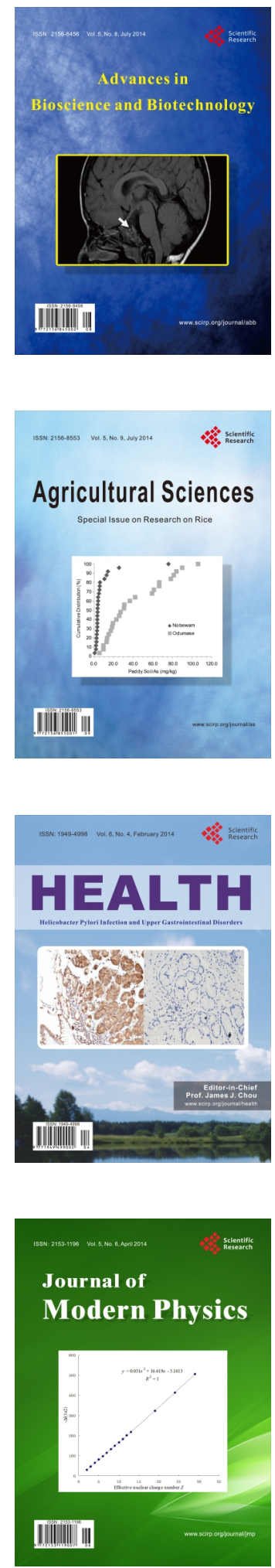
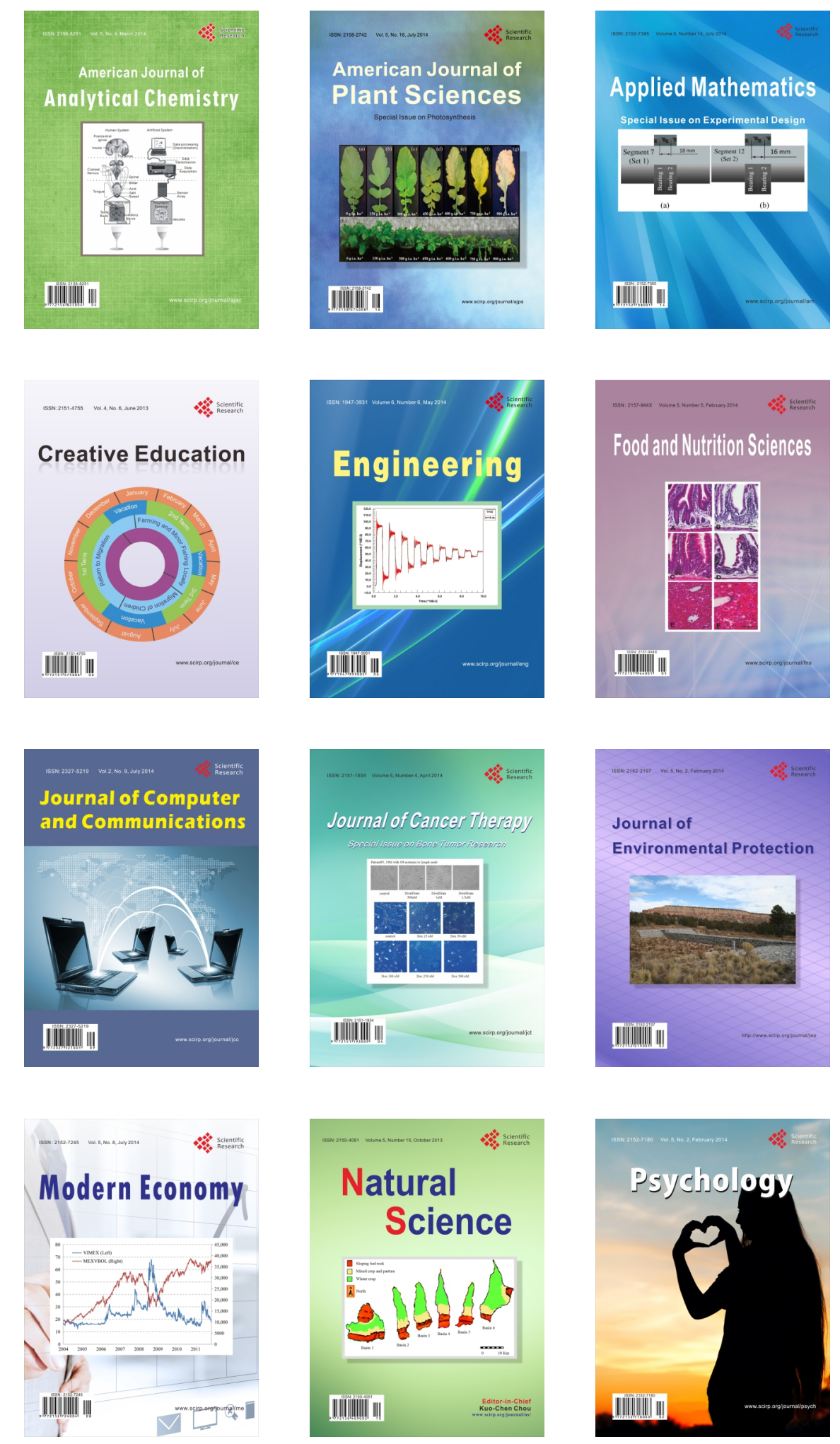